\title{
MiRNA-146a regulates the maturation and differentiation of vascular smooth muscle cells by targeting NF-кB expression
}

\author{
SHAOHONG DONG ${ }^{1}$, WEI XIONG ${ }^{1}$, JIANHUI YUAN ${ }^{2}$, JIANGHUA LI $^{1}$, JIANJUN LIU $^{2}$ and XINYUN XU ${ }^{2}$ \\ ${ }^{1}$ Department of Cardiology, The Second Clinical Medical College of Jinan University, Shenzhen People's Hospital, \\ Shenzhen, Guangdong 518020; ${ }^{2}$ Key Laboratory of Modern Toxicology of Shenzhen, \\ Shenzhen Center for Disease Control and Prevention, Shenzhen, Guangdong 518055, P.R. China
}

Received February 3, 2013; Accepted June 6, 2013

DOI: $10.3892 / \mathrm{mmr} .2013 .1538$

\begin{abstract}
Dysfunction of vascular smooth muscle cells (VSMCs) is key in the pathogenesis of proliferative cardiovascular diseases, including atherosclerosis and post-angioplasty restenosis. However, to date, the molecular mechanisms of this pathological process have not been elucidated. Growing evidence indicates that microRNAs (miRNAs) are a class of novel gene regulators. Recently, miR-146a was shown to be highly expressed in rat balloon-injured vascular walls as well as in peripheral blood mononuclear cells (PBMCs) from patients with acute coronary syndrome (ACS). The aim of the present study was to investigate the role of miR-146a in regulating VSMC fate and the possible underlying mechanisms involved. Our results revealed that the expression of miR-146a was increased in proliferative VSMCs. Subsequently, we observed that the knockdown of miR-146a significantly inhibited the proliferative and migratory properties of VSMCs in vitro, while it markedly promoted the apoptotic capacity of VSMCs. In addition, we demonstrated that the protein expression of nuclear factor- $\kappa \mathrm{Bp} 65(\mathrm{NF}-\kappa \mathrm{Bp} 65)$ and the proliferative cell nuclear antigen (PCNA), known as critical transcriptional factors, were downregulated. By contrast, the crucial proapoptotic molecule Bax has been revealed to be upregulated following miR-146a knockdown. These results support the conclusion that miR-146a is a novel regulator of VSMC fate and may be a new biomarker or therapeutic target for proliferative cardiovascular disease.
\end{abstract}

\section{Introduction}

It is well established that vascular smooth muscle cells (VSMCs) are not terminally differentiated (1). Their transi-

Correspondence to: Professor Shaohong Dong, Department of Cardiology, The Second Clinical Medical College of Jinan University, Shenzhen People's Hospital, No. 1017 Dongmen North Road, Shenzhen, Guangdong 518020, P.R. China

E-mail: dsh266@medmail.com.cn

Key words: vascular smooth muscle cell, miR-146a, proliferation, apoptosis, nuclear factor- $\kappa \mathrm{B}$ tion and maintenance from the differentiated state to the dedifferentiated phenotype occurs on accelerated proliferation, migration and synthesis of extracellular matrix (ECM) components. The phenotypic modulation of VSMCs plays a crucial role in the pathogenesis of a wide range of cardiovascular diseases, including atherosclerosis, in-stent restenosis and hypertension (2). However, despite advances in this research area, the molecular mechanisms underlying VSMC phenotypic modulation have not been completely understood.

MicroRNAs (miRNAs or miRs) are endogenous, small, single-stranded, 20 to 26 nucleotides in length, evolutionarily conserved RNAs. These noncoding RNAs, which have been shown to be ubiquitous in all eukaryotes, including vertebrates, negatively regulate more than one-third of protein-coding genes in the human genome by degrading or inhibiting their specific target genes at the post-transcriptional level (3-5). Their essential roles in physiological and pathophysiological processes, initially revealed in cancer, sepsis, viral infection and development, were further explored in cardiovascular diseases, including cardiogenesis, cardiac hypertrophy, arrhythmia, heart failure and cardiovascular remodeling (6-10). Recent studies have revealed that miR146a was aberrantly expressed and upregulated in rat arteries after balloon injury (11). Evidence has also demonstrated that miR-146a was involved in innate immunity and inflammatory responses (12). However, little is known about the effect of miR-146a on VSMC phenotypic modulation, as well as its molecular basis.

In the present study, we explored the possible role of miR-146a in the proliferative and migratory properties of VSMCs by transfecting small interfering RNA (siRNA) to knockdown miR-146a. Nuclear factor $\kappa$ Bp65 (NF- $\mathrm{Bp} 65)$, proliferating cell nuclear antigen (PCNA) and Bax, which are critical pro-inflammatory factors and transcriptional factors in the induction of vascular dysfunction and remodeling, were investigated in order to better understand the regulatory mechanisms of miR-146a that are responsible for regulating VSMC phenotypes.

\section{Materials and methods}

Cell culture. Primary VSMCs were obtained and isolated from the thoracic aortic media of male Sprague-Dawley 
rats (100-150 g) and were then propagated in high-glucose Dulbecco's modified Eagle's medium (DMEM) with 10\% heat-inactivated fetal bovine serum (FBS; purchased from Gibco-BRL, Carlsbad, CA, USA), $100 \mathrm{U} / \mathrm{ml}$ of penicillin and $100 \mu \mathrm{g} / \mathrm{ml}$ of streptomycin in a humidified incubator containing $5 \% \mathrm{CO}_{2}$ at $37^{\circ} \mathrm{C}$. Cells between passages 3 and 8 were used for the subsequent experiments. The study was approved by the Ethics Committee of Shenzhen People's Hospital, Shenzhen, China.

Quantification of miR-146a. To identify the potential role of miR-146a in VSMC biology, the expression signature of miR-146a in quiescent and proliferative cells was first surveyed by real-time PCR. After culture in various concentrations of FBS $(5,10,20 \%)$ for $48 \mathrm{~h}$, total RNA of VSMCs was extracted by using the RNeasy Mini kit (Qiagen, Hilden, Germany) and reverse transcribed to cDNA using the One Step PrimeScript miRNA cDNA Synthesis kit (Takara Bio, Inc., Shiga, Japan). Quantitative real-time PCR of miR-146a was accomplished by using the ABI PRISM 7900 Sequence Detection System (Applied Biosystems, Foster City, CA, USA) with the SYBR Premix Ex Taq kit (Takara Bio, Inc.). miR-146a was amplified using the PrimeScript Universal Primer (Takara Bio, Inc.) together with miRNA-specific primer (5'-TGAGAACTGAATTCCATGGGTT-3'). U6 RNA was used as an internal control. miRNA-146a-specific primer and U6 primers were synthesized by Sangon (Shanghai, China). The relative expression levels of miRNA-146a were calculated based on the following equation: relative miR-146a level = $2-(\Delta \mathrm{Ct}$ sample- $\Delta \mathrm{Ct}$ control $)$. All PCR was performed according to the manufacturer's instructions.

Knockdown of miR-146a. The antisense miR-146a oligonucleotide (miR-146a inhibitor) and the negative control miR-146a oligonucleotide (miR-146a control) were synthesized by GenePharma (Shanghai, China). The sequences of these oligonucleotides were as follows: 5'-AACCCAUGGAAUUCA GUUCUCA-3' (miR-146a inhibitor) and 5'-CAGUACUUU UGUGUAGUACAA-3' (miR-146a control). The miR-146a oligonucleotides contained 2/-O-methyl modifications at every base. At $24 \mathrm{~h}$ prior to transfection, VSMCs were cultured in DMEM without antibiotics and serum. The cells were classified into three groups: normal VSMCs, miR-146a control $(50 \mathrm{nM})$ and miR-146a inhibitor $(10,50,100 \mathrm{nM})$. All cells were transfected using Lipofectamine 2000 (Invitrogen Life Technologies, Carlsbad, CA, USA) according to the manufacturer's instructions. At $5 \mathrm{~h}$ after transfection, the supernatants were decanted and replaced by new DMEM containing $10 \%$ FBS. After incubation for $48 \mathrm{~h}$, relative expression of miR146a in different VSMCs was investigated by real-time PCR according to the methods described previously.

VSMC proliferation. VSMC proliferation was determined by cell counting and bromodeoxyuridine (BrdU) incorporation assay (Cell Proliferation ELISA; Roche, Basel, Switzerland). Briefly, VSMCs were seeded in a 96-well plate at a density of $5 \times 10^{3}$ cells per well for $24 \mathrm{~h}$. Cells were then transfected with miR-146a inhibitor (50 nM), miR-146a control (50 nM) or phosphate-buffered solution (PBS). After cell transfection, VSMCs were incubated in DMEM containing 10\% FBS for
12, 24 and 48 h. For cell counting, VSMCs were trypsinized and resuspended in PBS. The number of VSMCs was subsequently counted using a hemocytometer under a microscope. BrdU incorporation analysis was performed according to the manufacturer's instructions. The absorbance was measured at $450 \mathrm{~nm}$ with a reference wavelength at $690 \mathrm{~nm}$. Cell viability experiments were performed by trypan dye after BrdU treatment, and cell viability was always $>98 \%$. All experiments were performed in sextuplicate.

VSMC migration. The effect of miR-146a on VSMC migration was assessed using the Transwell system (Corning, NY, USA) with polycarbonate filters $(6.5-\mathrm{mm}$ diameter, $8.0-\mu \mathrm{m}$ pore size). At $48 \mathrm{~h}$ after treatment with PBS, miRNA-146a control or miRNA-146a inhibitor, VSMCs were harvested by trypsinization and resuspended. Cell suspensions at a density of $1 \times 10^{4}$ cells were added into the upper chambers of the wells in $200 \mu \mathrm{l}$ DMEM medium containing 5\% FBS and incubated for $12 \mathrm{~h}$, while the lower chambers were filled with DMEM medium containing 20\% FBS in order to induce cell migration. The VSMCs on the upper surface of the membrane were scraped softly. Cells that migrated to the lower surface were fixed with methanol and stained with $0.1 \%$ crystal violet dye. Cells were counted in 5 random views under a light microscope. Experiments were performed on 6 wells for each condition and repeated 3 times.

Flow cytometry. VSMC apoptosis was investigated by flow cytometry analysis with an Annexin V-PE/7-AAD Apoptosis Detection kit (KeyGEN Biotech, Nanjing, China). A total of $48 \mathrm{~h}$ after transfection in 6-well plates, VSMCs were harvested and washed twice in cold PBS, subsequently centrifuged and resuspended in $500 \mu \mathrm{l}$ binding buffer. Annexin V-PE $(1 \mu \mathrm{l})$ and 7-amino-actinomycin D (5 $\mu \mathrm{l})$ were successively added in order to stain the cells. The samples were measured by flow cytometry (BD Biosciences, Franklin Lakes, NJ, USA) according to the manufacturer's instructions. The apoptotic percentage was then calculated. Each group possessed 6 samples.

Western blot analysis. VSMCs treated with PBS, miR-146a control or miR-146a inhibitor were cultured in DMEM medium containing $10 \%$ FBS for $48 \mathrm{~h}$. Total protein $(20 \mu \mathrm{g})$ extracted from the cells was resolved on $10 \%$ SDS-polyacrylamide gels and electrotransferred onto nitrocellulose membranes by electrophoresis. The membranes were blocked in 5\% non-fat milk in TBS containing $0.3 \%$ Tween-20, then incubated overnight with polyclonal rabbit anti-rat NF- $\kappa$ Bp65 (1:1000 solution, Abcam, Cambridge, MA, USA), PCNA (1:500 solution, Santa Cruz Biotechnology, Santa Cruz, CA, USA) and Bax (1:500 solution, Santa Cruz Biotechnology). The blots were subsequently incubated in 1:6,000 dilutions of goat anti-rabbit horseradish peroxidase-conjugated secondary antibodies. Enhanced chemiluminescence (ECL) developing solution $(100 \mu \mathrm{l})$ was added in each sample to detect the protein signal with a Quant RT ECL cold CCD imaging system (General Electric, Fairfield, CT, USA).

Statistical analysis. All statistical analysis was performed with SPSS 16.0 (SPSS, Inc., Chicago, IL, USA). Data are 
presented as the means \pm standard error. For relative gene expression, the mean value of the vehicle group is defined as $100 \%$. Differences were compared by t-test and ANOVA. $\mathrm{P}<0.05$ was considered to indicate a statistically significant result. All experiments were performed at least three times.

\section{Results}

Relative expression of miR-146a in VSMCs. FBS is well acknowledged as a proproliferative factor. We first examined the expression of miR-146a in VSMCs cultured in different concentrations of FBS. The expression levels of miR-146a were increased by FBS in a concentration-dependent manner. The miR-146a level in VSMCs cultured with 20\% FBS was notably higher than that in VSMCs cultured with 5\% FBS $(\mathrm{P}<0.05$; Fig. 1). The significantly increased expression level of miR-146a in proliferative VSMCs indicates that miR-146a may promote the proliferation of VSMCs and reduce their apoptosis.

To knockdown miR-146a in vitro, we employed siRNAlabeled 6-carboxyfluorescein to treat VSMCs by using Lipofectamine 2000. As shown in Fig. 2A, extensive green fluorescence signals were observed in the cytoplasm of VSMCs after treatment for $5 \mathrm{~h}$. The average transfection efficiency exceeded $90 \%$. Subsequently, we investigated the relative expression of miR-146a in cultured VSMCs after $48 \mathrm{~h}$ of treatment by PBS, miR-146a control or miR-146a inhibitor. As shown in Fig. 2B, the expression of miR-146a in miR-146a inhibitor-treated VSMCs was significantly lower than that in PBS-treated and miR-146a control-treated VSMCs $(\mathrm{P}<0.05)$. The dose-dependent response of miR-146a expression to the inhibitor was identified after treatment with various concentrations of miR-146a inhibitors. Fig. 2C shows that expression of miR-146a was downregulated by inhibitors in a dose-dependent manner $(\mathrm{P}<0.05)$.

Knockdown of miR-146a inhibited proliferation of VSMCs. In subsequent experiments, we identified the role of miR-146a in the proliferation of VSMCs by applying two different methods: cell counting and BrdU incorporation assay. As shown in Fig. 3, the cell number and BrdU ELISA absorbance showed no significant differences among VSMCs treated with PBS, miR-146a control and miR-146a inhibitor, respectively, prior to transfection $(\mathrm{P}>0.05)$. However, after transfection, the VSMCs treated with miR-146a inhibitor exhibited markedly lowered ability for proliferation compared with the VSMCs treated with PBS or miR-146a control. These differences were observed from 12 to $48 \mathrm{~h}$ after treatment (all $\mathrm{P}<0.05)$. These results indicated that miR-146a is a proproliferative regulator for VSMCs.

Knockdown of miR-146a inhibits migration of VSMCs. To further confirm that knockdown of miR-146a is capable of inhibiting VSMC migration, the Transwell system was utilized. As expected, the number of cells that migrated to the lower chamber in VSMCs treated with miR-146a inhibitor for $48 \mathrm{~h}$ was markedly reduced compared with those in VSMCs treated with PBS or miR-146a control ( $\mathrm{P}<0.01$; Fig. 4). These results indicate that miR-146a is capable of promoting migration of VSMCs in vitro.

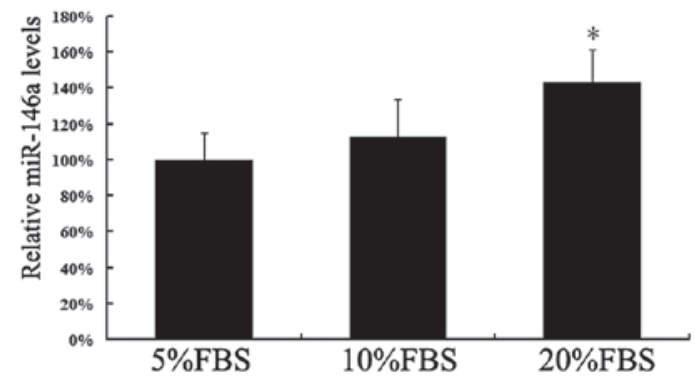

Figure 1. VSMCs were cultured in various concentrations of fetal bovine serum for $48 \mathrm{~h}$ and miR-146a expression was identified by real-time PCR. ${ }^{*} \mathrm{P}<0.05$ compared with $5 \%$ fetal bovine serum cultured VSMCs. FBS, fetal bovine serum; VSMCs, vascular smooth muscle cells.

A

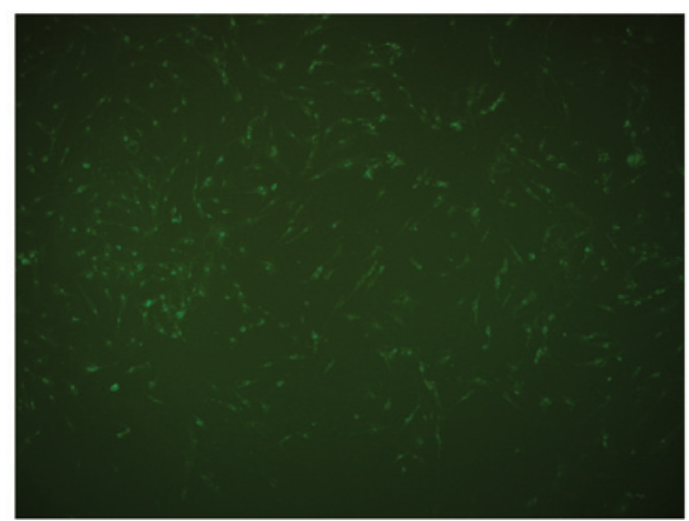

B

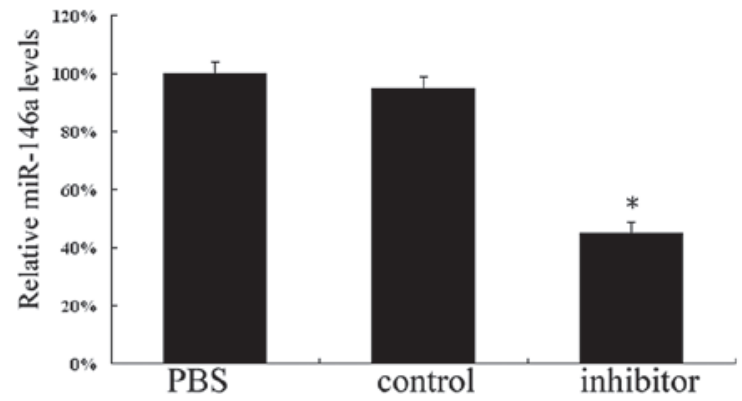

C

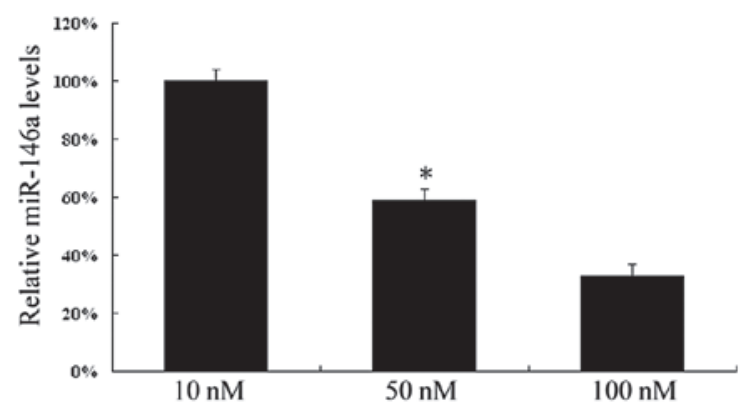

Figure 2. Expression of miR-146a inhibitor in cultured VSMCs. (A) FAM fluorescence signals in the cytoplasm of cells after treatment for $5 \mathrm{~h}$ (green; original magnification, x200). (B and C) The relative mRNA of miRNA-146a was assayed by real-time PCR. (B) The transcript level was significantly decreased in inhibitor-treated cells compared with that in PBS or controltreated cells. (C) Inhibitors caused a dose-dependent decrease in miR-146a expression. ${ }^{*} \mathrm{P}<0.05$ compared with other groups. VSMCs, vascular smooth muscle cells.

Knockdown of miR-146a promotes apoptosis of VSMCs. To explore whether miR-146a affects VSMC survival in vitro, we applied flow cytometry to examine the apoptotic aspects in 
A

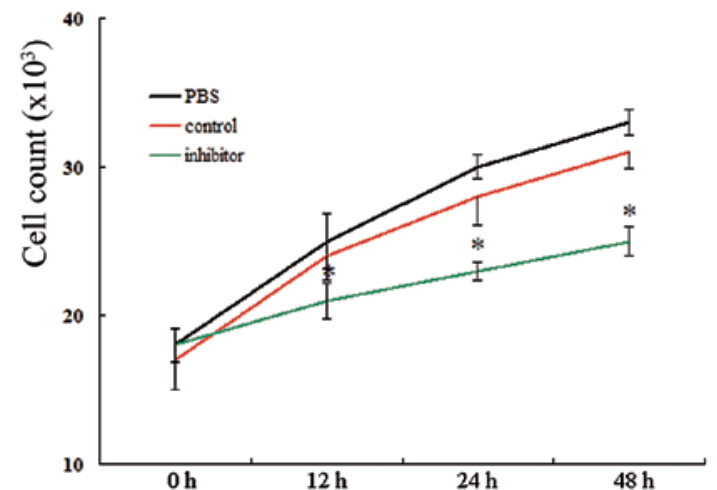

B

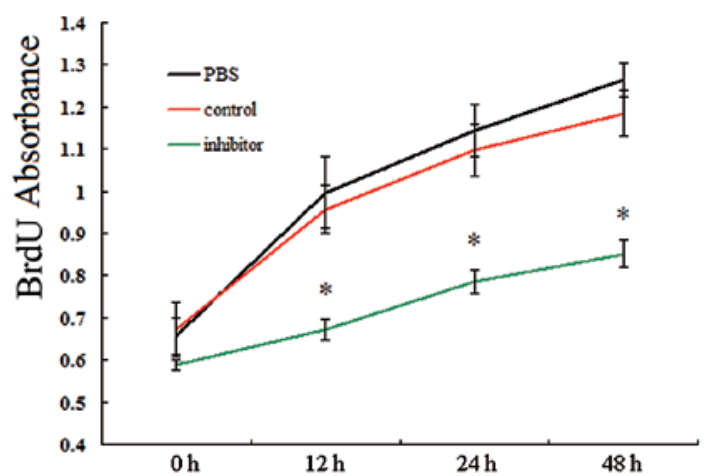

Figure 3. Effect of miR-146a inhibitor on proliferation in cultured VSMCs. (A) The cell counting and (B) BrdU incorporation assay in miR-146a inhibitor-treated VSMCs showed no marked differences prior to transfection $(0 \mathrm{~h})$ compared with other groups. After treatment with miR-146a inhibitor, cell number and BrdU ELISA absorbance were both significantly reduced from 12 to 48 h. $\mathrm{P}<0.05$ compared with PBS and control groups. VSMCs, vascular smooth muscle cells; BrdU, bromodeoxyuridine.

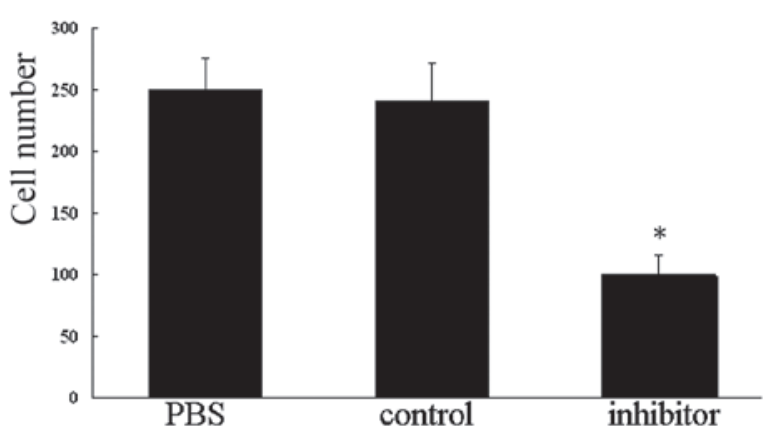

Figure 4. Effect of miR-146a knockdown on migration in cultured VSMCs. After treatment with PBS, miR-146a control or miR-146a inhibitor for $48 \mathrm{~h}$, VSMCs that migrated to the lower chamber were stained by crystal violet and counted. "P<0.01 compared with PBS and control groups. VSMCs, vascular smooth muscle cells; PBS, phosphate-buffered solution.

VSMCs after treatment for $48 \mathrm{~h}$. Combining Annexin V-PE with 7-AAD, three categories of cellular populations were detected: viable, early apoptotic, late apoptotic or necrotic cells. As shown in Fig. 5, the percentage of apoptotic cells in the miR-146a-inhibitor treated group was significantly increased compared with VSMCs treated with PBS or miR-146a control $(\mathrm{P}<0.05)$. These results indicated that knockdown of miR-146a is capable of promoting apoptosis of VSMCs in vitro and also supported the conclusion that knockdown of miR-146a inhibits VSMC proliferation.
Western blot analysis. To identify the potential molecular mechanisms underlying the regulation of proliferation and apoptosis by miR-146a in cultured VSMCs, the protein levels of NF- $\kappa \mathrm{Bp} 65$, PCNA and Bax were determined by western blot analysis. As shown in Fig. 6, the crucial transcriptional factor NF- $\mathrm{kBp} 65$ and the proliferative protein PCNA were confirmed to be downregulated in miR-146a inhibitor-treated VSMCs (both $\mathrm{P}<0.05$ ), while the important proapoptotic protein Bax was shown to be upregulated in VSMCs following miR-146a transfection $(\mathrm{P}<0.05)$.

\section{Discussion}

The emergence of miRNAs has greatly increased our knowledge of the molecular mechanisms underlying gene expression and regulation. Studies have identified important functions of miRNAs for the normal development and physiological homeostasis of organisms. Increasing evidence has also highlighted their potential roles as biomarkers in diagnosis and as targets for therapy (13-16). However, there remain a number of unclear details in the understanding of these complex gene regulatory networks.

Previously identified as a novel determinant of the molecular circuitry that governs innate immunity and inflammatory responses, miR-146a was initially investigated in the immune system, cancer and viral infections (12,17-23). It has been observed that miR-146a was upregulated in THP-1 cells, macrophages, Langerhans cells and monocytes after being induced by lipopolysaccharide (LPS), TNF- $\alpha$, IL-1 $\beta$ or TGF- $\beta 1(12,18,24)$. Similar outcomes were revealed in peripheral blood mononuclear cells (PBMCs) and synovial tissue from patients with rheumatoid arthritis $(25,26)$. In the majority of cells, these pro-inflammatory cytokines stimulated expression of miR-146a and are dependent on the NF- $\mathrm{KB}$ signaling pathway, with the exception of human lung alveolar epithelial cells (27). Notably, two critical downstream molecules of cytokine and Toll-like receptors (TLRs), TNF receptor-associated factor 6 (TRAF6) and IL-1 receptor-associated kinase 1 (IRAK1), were ascertained as direct target genes of miR-146 $(12,21)$. The activation of NF- $\mathrm{KB}$ is downregulated by miR-146 through a negative feedback regulatory loop involving TRAF6 and IRAK1. Recent evidence indicated that miRNAs may play a role in phenotypic modulation of VSMCs $(28,29)$. More importantly, enhanced expression of miR-146a was confirmed in the vascular wall after angioplasty and in PBMCs of patients with acute coronary syndrome (ACS) (11,30). The development of proliferative vascular diseases is an exquisitely orchestrated process that involves a cascade of inflammatory and immune responses, and subsequent activation, proliferation and migration of VSMCs, as well as accelerated synthesis of ECM components and eventual formation of neointimal lesions. These aforementioned studies suggested that miR-146a may play an important role in controlling VSMC fate.

In our present study, we first explored the role of miR-146a in the proliferation process of VSMCs. A significantly higher expression level of miR-146a was observed in proliferative VSMCs, which indicated that miR-146a is a proproliferative factor for VSMCs. To further verify the role of miR-146a in VSMC proliferation and migration, we employed the well established method of RNA interference to specifically knock- 
A

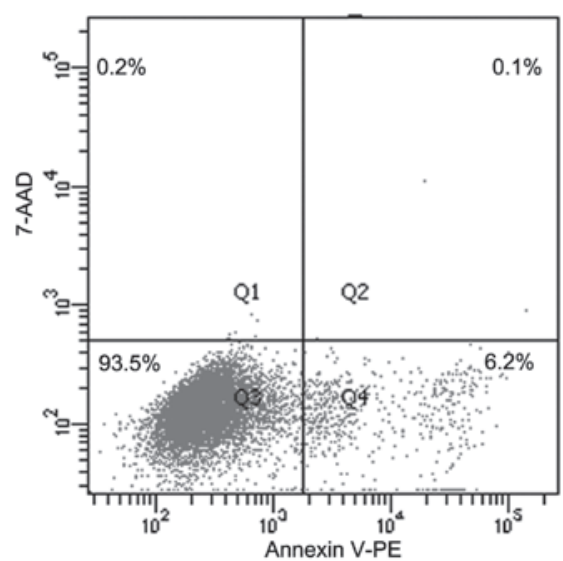

$\mathbf{C}$

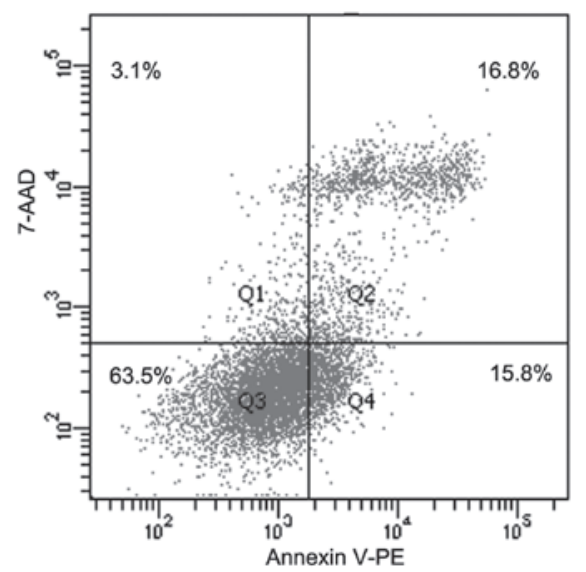

B

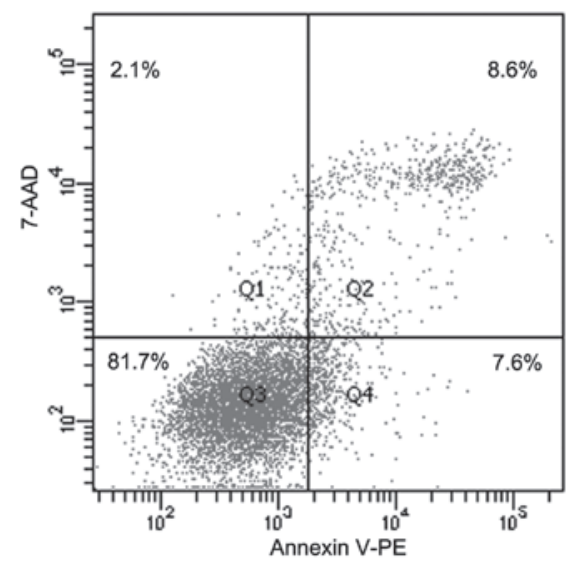

D

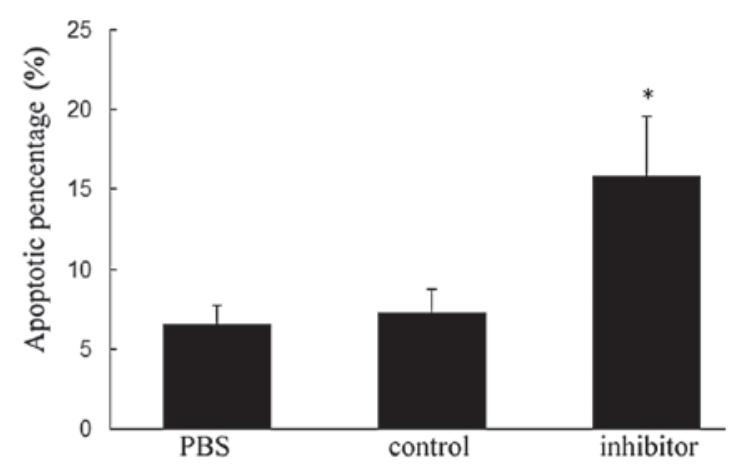

Figure 5. Effect of miR-146a knockdown on apoptosis in cultured VSMCs. Apoptosis of cultured VSMCs treated by (A) PBS, (B) miR-146a control or (C) miR-146a inhibitor was determined by flow cytometry combined with Annexin V-PE and 7-AAD. (D) Percentage of apoptotic cells in each group. ${ }^{*}$ < 0.05 compared with PBS and control groups. VSMCs, vascular smooth muscle cells; PBS, phosphate-buffered solution.
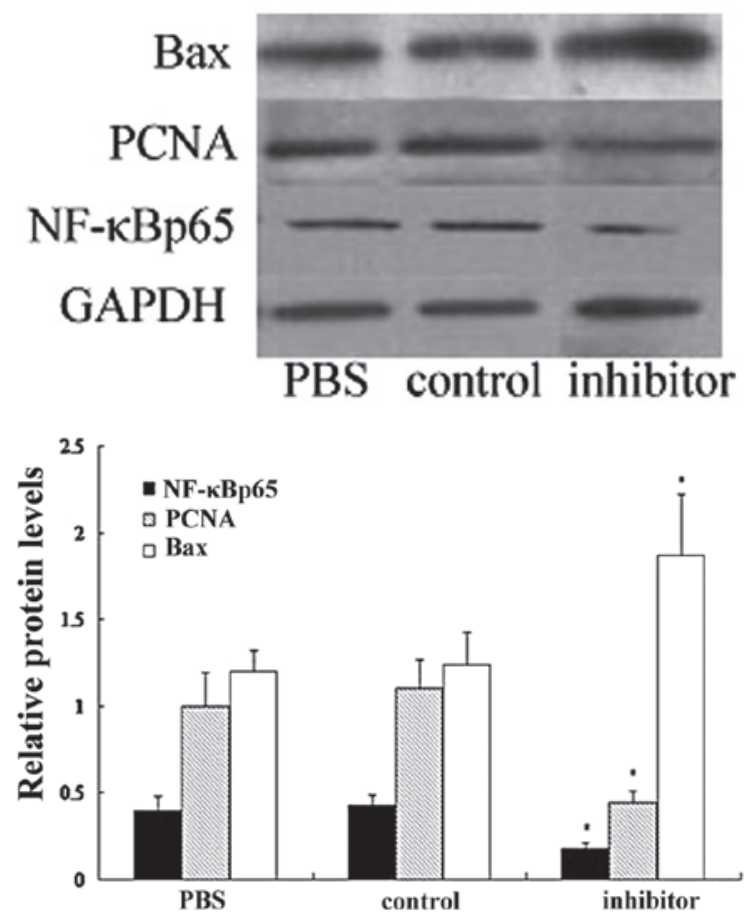

Figure 6. Effect of miR-146a knockdown on protein levels in cultured VSMCs. The protein levels of NF- $\mathrm{kBp} 65$ and PCNA were reduced by miR-146a inhibitor, while the protein level of Bax was enhanced by miR-146a inhibitor. ${ }^{*} \mathrm{P}<0.05$ compared with PBS and control groups. VSMCs, vascular smooth muscle cells; PCNA, proliferative cell nuclear antigen. down miR-146a in cultured VSMCs. The results revealed that miR-146a-specific siRNA was sufficient to inhibit the proliferative and migratory capacity of VSMCs, which indicated that miR-146a is an essential pro-proliferative and pro-migratory regulator for VSMCs. In addition, cell viability experiments determined by BrdU incorporation assay showed there was no nonspecific toxicity after miR-146a inhibitor delivery, which demonstrated that the effect of miR-146a siRNA in regulating VSMC fate is miR-146a specific.

The equilibrium between proliferation and apoptosis in VSMCs is responsible for neointimal fate. The increased VSMC proliferation or decreased VSMC apoptosis contribute to neointimal hyperplasia and vascular remodeling, which constitute the important factors in the pathogenesis of proliferative vascular diseases. To characterize the effect of miR-146a on VSMC functional modulation, the apoptotic assays were performed. We observed that exogenous inhibition of miR-146a markedly enhanced VSMC apoptosis. Moreover, western blot analysis revealed that the Bax protein, which is a key proapoptotic signal molecule (31), was simultaneously activated and upregulated in VSMCs transfected with miR-146a inhibitor, although the detailed mechanisms involved remain unclear. The anti-apoptotic effect of miR-146a is consistent with its pro-proliferative effect on VSMCs.

Our results from another western blot analysis also revealed that $\mathrm{NF}-\kappa \mathrm{Bp} 65$ and PCNA were involved in miR-146a-mediated VSMC maturation and differentiation. 
Notably, in the majority of immune and cancer cells, miR146a regulated the $\mathrm{NF}-\kappa \mathrm{B}$ signal pathway in a negative feedback manner $(12,21)$, while in our present study, the activity of NF- $\kappa$ Bp65 in VSMCs was markedly reduced by miR-146a inhibitor, which indicates that miR-146a stimulated activation of $\mathrm{NF}-\kappa \mathrm{B}$ in VSMCs. Similar upregulation of NF- $\kappa \mathrm{B}$ induced by miR-146a was observed in PMBCs from patients with ACS (30). It appears that miR-146a plays different roles in diverse cells due to its cell-type specificity. It is possible that miR-146a behaves as a tuning mechanism to prevent overstimulated inflammatory responses in the innate immune system, while in proliferative vascular diseases, including atherosclerosis, post-angioplasty restenosis and stroke, miR-146a acts as a promoter to play a role in the pathological process at an early stage.

In conclusion, we revealed that the noncoding small miRNA, miR-146a, is a novel regulator of VSMC proliferation, migration and apoptosis. The modulation of VSMC fate mediated by miR-146a was likely to be related to the activation of the NF- $\kappa \mathrm{B}$ signal transduction pathway. However, our work was only performed in loss-of-function experiments of miR-146a, therefore, further gain-of-function studies such as overexpression of miR-146a using its precursor or a viral vector should be implemented in order to clearly understand its mechanisms in regulating gene expression and to evaluate its potential as a biomarker or therapeutic target in proliferative cardiovascular diseases.

\section{Acknowledgements}

This study was supported by the Science Foundation of Shenzhen (Project number: 201102158).

\section{References}

1. Owens GK, Kumar MS and Wamhoff BR: Molecular regulation of vascular smooth muscle cell differentiation in development and disease. Physiol Rev 84: 767-801, 2004

2. Rzucidlo EM, Martin KA and Powell RJ: Regulation of vascular smooth muscle cell differentiation. J Vasc Surg 45: A25-A32, 2007.

3. Reinhart BJ, Slack FJ, Basson M, Pasquinelli AE, Bettinger JC, Rougvie AE, Horvitz HR and Ruvkun G: The 21-nucleotide let-7 RNA regulates developmental timing in Caenorhabditis elegans. Nature 403: 901-906, 2000.

4. Pasquinelli AE, Reinhart BJ, Slack F, Martindale MQ, Kuroda MI, Maller B, Hayward DC, Ball EE, Degnan B, Müller P, Spring J, Srinivasan A, Fishman M, Finnerty J, Corbo J, Levine M, Leahy P, Davidson E and Ruvkun G: Conservation of the sequence and temporal expression of let-7 heterochronic regulatory RNA. Nature 408: 86-89, 2000.

5. Lee RC and Ambros V: An extensive class of small RNAs in Caenorhabditis elegans. Science 294: 862-864, 2001.

6. Zhao Y, Ransom JF, Li A, Vedantham V, von Drehle M, Muth AN, Tsuchihashi T, McManus MT, Schwartz RJ and Srivastava D: Serum response factor regulates a muscle-specific microRNA that targets Hand2 during cardiogenesis. Nature 436: 214-220, 2005.

7. Zhao Y, Ransom JF, Li A, Vedantham V, von Drehle M, Muth AN, Tsuchihashi T, McManus MT, Schwartz RJ and Srivastava D: Dysregulation of cardiogenesis, cardiac conduction, and cell cycle in mice lacking miRNA-1-2. Cell 129: 303-317, 2007

8. Sayed D, Hong C, Chen IY, Lypowy J and Abdellatif M: MicroRNAs play an essential role in the development of cardiac hypertrophy. Circ Res 100: 416-424, 2007.

9. Yang B, Lin H, Xiao J, Lu Y, Luo X, Li B, Zhang Y, Xu C, Bai Y, Wang $\mathrm{H}$, Chen $\mathrm{G}$ and Wang Z: The muscle-specific microRNA miR-1 regulates cardiac arrhythmogenic potential by targeting GJA1 and KCNJ2. Nat Med 13: 486-491, 2007.
10. Yang B, Lin H, Xiao J, Lu Y, Luo X, Li B, Zhang Y, Xu C, Bai Y, Wang H, Chen $\mathrm{G}$ and Wang Z: MicroRNAs in the human heart: a clue to fetal gene reprogramming in heart failure. Circulation 116: 258-267, 2007

11. Ji R, Cheng Y, Yue J, Yang J, Liu X, Chen H, Dean DB and Zhang C: MicroRNA expression signature and antisense-mediated depletion reveal an essential role of microRNA in vascular neointimal lesion formation. Circ Res 100: 1579-1588, 2007.

12. Taganov KD, Boldin MP, Chang KJ and Baltimore D: NF- $\kappa B$ dependent induction of microRNA miR-146a, an inhibitor targeted to signaling proteins of innate immune responses. Proc Natl Acad Sci USA 103: 12481-12486, 2006.

13. Bandiera S, Hatem E, Lyonnet S and Henrion-Caude A: microRNAs in diseases: from candidate to modifier genes. Clin Genet 77: 306-313, 2010.

14. Esteller M: Non-coding RNAs in human disease. Nat Rev Genet 12: 861-874, 2011.

15. Kaikkonen MU, Lam MT and Glass CK: Non-coding RNAs as regulators of gene expression and epigenetics. Cardiovasc Res 90: 430-440, 2011.

16. Fichtlscherer S, Zeiher AM and Dimmeler S: Circulating microRNAs: biomarkers or mediators of cardiovascular diseases? Arterioscler Thromb Vasc Biol 31: 2383-2390, 2011.

17. Nahid MA, Pauley KM, Satoh M and Chan EK: miR-146a is critical for endotoxin-induced tolerance: implication in innate immunity. J Biol Chem 284: 34590-34599, 2009.

18. Jurkin J, Schichl YM, Koeffel R, Bauer T, Richter S, Konradi S, Gesslbauer B and Strobl H: miR-146a is differentially expressed by myeloid dendritic cell subsets and desensitizes cells to TLR2dependent activation. J Immunol 184: 4955-4965, 2010.

19. Lu LF, Boldin MP, Chaudhry A, Lin LL, Taganov KD, Hanada T, Yoshimura A, Baltimore D and Rudensky AY: Function of miR-146a in controlling treg cell-mediated regulation of Th1 responses. Cell 142: 914-929, 2010.

20. He H, Jazdzewski K, Li W, Liyanarachchi S, Nagy R, Volinia S, Calin GA, Liu CG, Franssila K, Suster S, Kloos RT, Croce CM and de la Chapelle A: The role of microRNA genes in papillary thyroid carcinoma. Proc Natl Acad Sci USA 102: 19075-19080, 2005.

21. Bhaumik D, Scott GK, Schokrpur S, Patil CK, Campisi J and Benz CC: Expression of microRNA-146 suppresses NF- $\kappa$ B activity with reduction of metastatic potential in breast cancer cells. Oncogene 27: 5643-5647, 2008.

22. Li Y, Vandenboom TG 2nd, Wang Z, Kong D, Ali S, Philip PA and Sarkar FH: miR-146a suppresses invasion of pancreatic cancer cells. Cancer Res 70: 1486-1495, 2010.

23. Cameron JE, Yin Q, Fewell C, Lacey M, McBride J, Wang X, Lin Z, Schaefer BC and Flemington EK: Epstein-Barr virus latent membrane protein 1 induces cellular microRNA miR-146a, a modulator of lymphocyte signaling pathways. J Virol 82: 1946-1958, 2008.

24. Liu G, Friggeri A, Yang Y, Park YJ, Tsuruta Y and Abraham E: miR-147, a microRNA that is induced upon toll-like receptor stimulation, regulates murine macrophage inflammatory responses. Proc Natl Acad Sci USA 106: 15819-15824, 2009.

25. Pauley KM, Satoh M, Chan AL, Bubb MR, Reeves WH and Chan EK: Upregulated miR-146a expression in peripheral blood mononuclear cells from rheumatoid arthritis patients. Arthritis Res Ther 10: R101, 2008.

26. Nakasa T, Miyaki S, Okubo A, Hashimoto M, Nishida K, Ochi M and Asahara H: Expression of microRNA-146 in rheumatoid arthritis synovial tissue. Arthritis Rheum 58: 1284-1292, 2008.

27. Perry MM, Moschos SA, Williams AE, Shepherd NJ, LarnerSvensson HM and Lindsay MA: Rapid changes in microrna-146a expression negatively regulate the IL- $1 \beta$-induced inflammatory response in human lung alveolar epithelial cells1. J Immunol 180: 5689-5698, 2008.

28. Cordes KR, Sheehy NT, White MP, Berry EC, Morton SU, Muth AN, Lee TH, Miano JM, Ivey KN and Srivastava D: miR-145 and miR-143 regulate smooth muscle cell fate and plasticity. Nature 460: 705-710, 2009.

29. Davis BN, Hilyard AC, Nguyen PH, Lagna G and Hata A: Induction of microRNA-221 by platelet-derived growth factor signaling is critical for modulation of vascular smooth muscle phenotype. J Biol Chem 284: 3728-3738, 2009.

30. Guo M, Mao X, Ji Q, Lang M, Li S, Peng Y, Zhou W, Xiong B and Zeng Q: miR-146a in PBMCs modulates Th1 function in patients with acute coronary syndrome. Immunol Cell Biol 88: 555-564, 2010.

31. Lalier L, Cartron PF, Juin P, Nedelkina S, Manon S, Bechinger B and Vallette FM: Bax activation and mitochondrial insertion during apoptosis. Apoptosis 12: 887-896, 2007. 